\title{
Do thermal fluctuations influence the recoil loops of nanocomposite magnets?
}

\author{
Chuan-bing Rong, ${ }^{\text {a) }}$ Yuzi Liu, and J. Ping Liu ${ }^{\text {b) }}$ \\ Department of Physics, The University of Texas at Arlington, Arlington, Texas 76019, USA
}

(Received 27 February 2008; accepted 17 June 2008; published online 29 July 2008)

\begin{abstract}
An exchange-coupled $\mathrm{FePt} / \mathrm{Fe}_{3} \mathrm{Pt}$ nanocomposite system with well defined hard/soft two-phase morphology was prepared by reducing chemically synthesized $\mathrm{FePt} / \mathrm{Fe}_{3} \mathrm{O}_{4}$ bimagnetic nanoparticles. It was interesting to observe that the recoil loop openness is strongly dependent on the sweep rate of applied fields. A quantitative analysis shows a linear relation between the openness of recoil loops and the reciprocal activation volume, indicating that the recoil loops are intimately related to thermal fluctuation. The large open area of the recoil loops for nanocomposite magnets compared to that of single phase magnets is attributed to the unstable magnetic moments in the soft phase. (C) 2008 American Institute of Physics. [DOI: 10.1063/1.2956392]
\end{abstract}

Exchange-coupled nanocomposite magnets, which combine the high magnetocrystalline anisotropy of a hard magnetic phase and the high magnetization of a soft magnetic phase, have attracted tremendous attention due the high potential energy product $(\mathrm{BH})_{\max }$ above $100 \mathrm{MGOe}^{1-4}$ One of the key issues to the high energy products in the nanocomposite magnets is the effective exchange coupling between the two phases, which can be influenced by many factors such as microstructure, magnetocrystalline anisotropy, and the interphase exchange stiffness. Understanding the magnetization behavior of the nanocomposite magnets then will give feedback to optimize the materials design. One of the most effective ways to characterize the exchange-coupling strength in nanostructured permanent magnets is to measure recoil loops of the nanocomposites. ${ }^{5-11}$ It is commonly accepted that the recoil loops are wide open for hard/soft nanocomposite magnets but are narrow and even closed for a single-phase magnet. ${ }^{5-8,11}$ However, the mechanism of the open recoil loops is still a subject of debate, even though it is widely agreed that it is related to the soft phase properties in the nanocomposites. ${ }^{5,6,8,12}$ In our investigation, it has been found that the openness of the recoil loops of FePt-based nanocrystalline magnets is strongly dependent on the sweep rate of applied magnetic fields, especially for the nanocomposite-type $\mathrm{FePt} / \mathrm{Fe}_{3} \mathrm{Pt}$ magnets. More interestingly, a linear correlation has been observed between the openness of the recoil loops and the reciprocal activation volume, indicating that the origin of the open recoil loops is related to thermal fluctuations.

$\mathrm{FePt} / \mathrm{Fe}_{3} \mathrm{Pt}$ nanocomposites were obtained by annealing bimagnetic $\mathrm{FePt} / \mathrm{Fe}_{3} \mathrm{O}_{4}$ bricklike nanoparticles under forming gas $\left(\mathrm{Ar}+7 \% \mathrm{H}_{2}\right)$ at different temperatures $\left(T_{a}\right.$ $=400-800{ }^{\circ} \mathrm{C}$ ) for $1 \mathrm{~h}^{13}$ The crystalline structure and morphology were characterized by $\mathrm{x}$-ray diffraction (XRD) and transmission electron microscopy (TEM). The overall Fe:Pt atomic ratio of the as-synthesized and annealed samples, which was determined by energy dispersive $\mathrm{x}$-ray (EDX) analysis, is around $(63 \pm 2):(37 \pm 2)$. The time-dependent recoil loops were measured by an alternating gradient magne-

\footnotetext{
${ }^{a)}$ Electronic mail: crong@uta.edu.

${ }^{b)}$ Electronic mail: pliu@uta.edu.
}

tometer (AGM) using different sweep rates of the applied field. The low-temperature magnetic properties were studied by a superconducting quantum interference device (SQUID) magnetometer.

The high-resolution TEM images (not shown here) confirmed that the as-synthesized particles are bimagnetic bricklike nanoparticles about $14 \mathrm{~nm}$ in total length. One-half of each brick $(7 \mathrm{~nm})$ is composed of fcc FePt cubic particles, and the other half is $\mathrm{Fe}_{3} \mathrm{O}_{4}$ cubic particles. Figure 1 shows the TEM images of the 650 and $800{ }^{\circ} \mathrm{C}$ annealed samples. It was found that the grain size of the $650{ }^{\circ} \mathrm{C}$ annealed sample is only $8-13 \mathrm{~nm}$, while serious grain growth was observed in the $800{ }^{\circ} \mathrm{C}$ annealed sample. The insets compare the selected area electron diffraction (SAED) patterns of the 650 and $800{ }^{\circ} \mathrm{C}$ annealed samples. One can see that the $\mathrm{Fe}_{3} \mathrm{Pt}$ phase forms during reductive annealing at $650{ }^{\circ} \mathrm{C}$ while the $800{ }^{\circ} \mathrm{C}$ annealed sample consists of only the $L 1_{0} \mathrm{FePt}$ phase. The change from the two-phase structure to single-phase structure with increasing annealing temperature may be related to the enhanced atomic interdiffusion at high temperatures that leads to the formation of the $L 1_{0}$ phase with high Fe contents. ${ }^{11,14}$ It should be noted that the Rietveld refinement of XRD patterns confirms that the volume fraction of the $\mathrm{Fe}_{3} \mathrm{Pt}$ phase decreases from $28 \pm 3 \%$ to $8 \pm 2 \%$ with increasing annealing temperature from 500 to $650{ }^{\circ} \mathrm{C}$.

To measure the recoil loops, we set the basic sweep rate of the applied field as $\eta_{0}=10^{4} \mathrm{Oe} / \mathrm{s}$, which is the limit of the AGM. Figure 2 gives the major and recoil loops of the 550

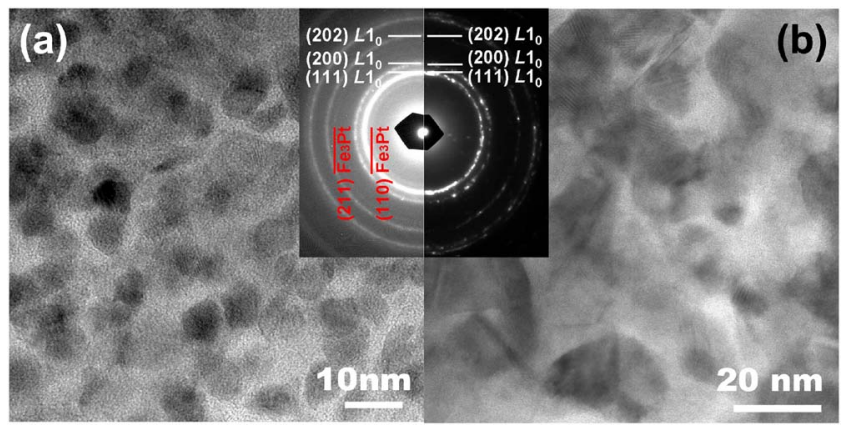

FIG. 1. (Color online) TEM images of the (a) $650{ }^{\circ} \mathrm{C}$ and (b) $800{ }^{\circ} \mathrm{C}$ annealed samples. The insets are the SAED patterns of 650 and $800^{\circ} \mathrm{C}$ annealed samples, respectively. 


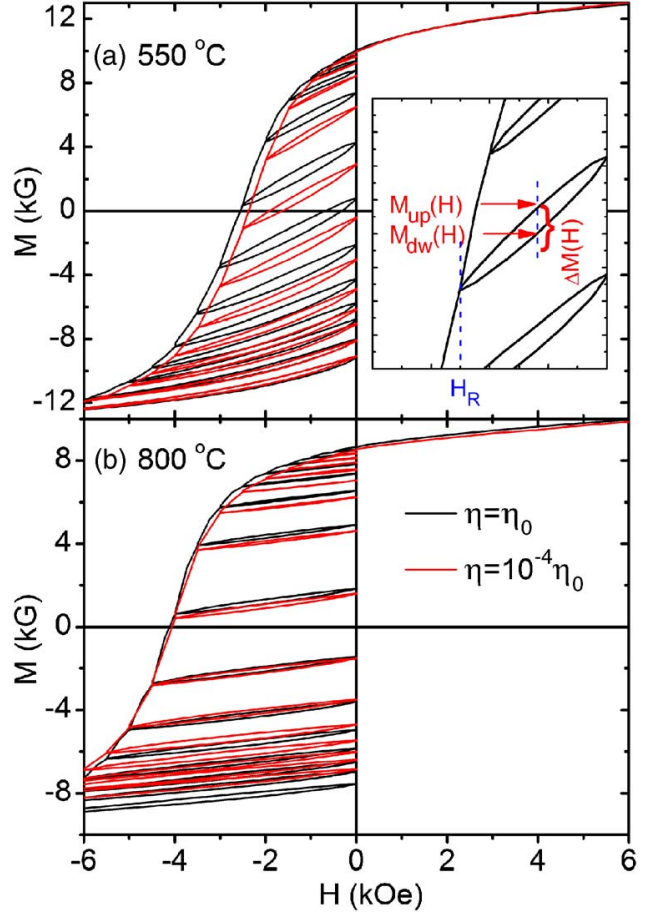

FIG. 2. (Color online) Recoil loops of the (a) 550 and (b) $800{ }^{\circ} \mathrm{C}$ annealed samples with different sweep rates: (black) $\eta=\eta_{0}$ and (red) $\eta=10^{-4} \eta_{0}$. The inset defines $M_{\mathrm{up}}(H), M_{\mathrm{dw}}(H)$, and $\Delta M_{\mathrm{rc}}(H)$.

and $800{ }^{\circ} \mathrm{C}$ annealed samples with $\eta=\eta_{0}$ and $10^{-4} \eta_{0}$. It is seen that open recoil loops were observed for both samples. However, the open areas of the $550{ }^{\circ} \mathrm{C}$ annealed sample is larger than that of the $800{ }^{\circ} \mathrm{C}$ annealed sample. More interestingly, the open areas of the fast measurements are larger than those of the slow ones for the $550{ }^{\circ} \mathrm{C}$ annealed sample, indicating that the openness of the recoil loops is related to the field sweep rate. To quantitatively characterize the openness of recoil loops, we use the normalized ratio $\Delta m_{\mathrm{rc}}(H)$ $=\left[M_{\text {up }}(H)-M_{\mathrm{dw}}(H)\right] / M_{s}$ where $M_{\text {up }}(H)$ and $M_{\mathrm{dw}}(H)$ are the magnetizations of the upper and lower branches, respectively, on the recoil loops under an applied field $H$ [see Fig. 2(b)]. $M_{s}$ is the saturated magnetization. The maximum $\Delta m_{\mathrm{rc}}(H)$ in each recoil loop (with a maximum applied reversal field $\left.H_{R}\right)$ was then defined as $\Delta m_{\mathrm{rc}}^{m}\left(H_{R}\right)$.

Figure 3 shows the dependence of $\Delta m_{\mathrm{rc}}^{m}\left(H_{R}\right)$ on $H_{R}$ for the $550,600,650$, and $800{ }^{\circ} \mathrm{C}$ annealed samples. Peaks of $\Delta m_{\mathrm{rc}}^{m}\left(H_{R}\right)$ were observed for the $550-650{ }^{\circ} \mathrm{C}$ annealed samples, while there is no peak for the $800{ }^{\circ} \mathrm{C}$ annealed sample. The appearance of the peaks and the applied fields at which the peak appeared may be related to the magnetization behavior of the soft magnetic phase. Here, we define the peak value on each $\Delta m_{\mathrm{rc}}^{m}\left(H_{R}\right)$ curve as $\Delta m_{\mathrm{rc}}^{\mathrm{pm}}$. The inset of Fig. 3 gives the dependence of $\Delta m_{\mathrm{rc}}^{\mathrm{pm}}$ with $\eta=\eta_{0}$ on annealing temperature. One can see that $\Delta m_{\mathrm{rc}}^{\mathrm{pm}}$ decreases fast with increasing annealing temperature up to $650{ }^{\circ} \mathrm{C}$ due to the decrease of soft-phase content. Higher annealing temperature leads to the disappearance of the soft phase and thus a small change in $\Delta m_{\mathrm{rc}}^{\mathrm{pm}}$. This is consistent with the previous report that the recoil loop openness is related to the two-phase structure. . $^{5,11}$

Interestingly, it was also found that $\Delta m_{\mathrm{rc}}^{m}\left(H_{R}\right)$ decreases significantly with decreasing $\eta$ for the $550-650{ }^{\circ} \mathrm{C}$ annealed samples, while it is not obvious for those annealed at

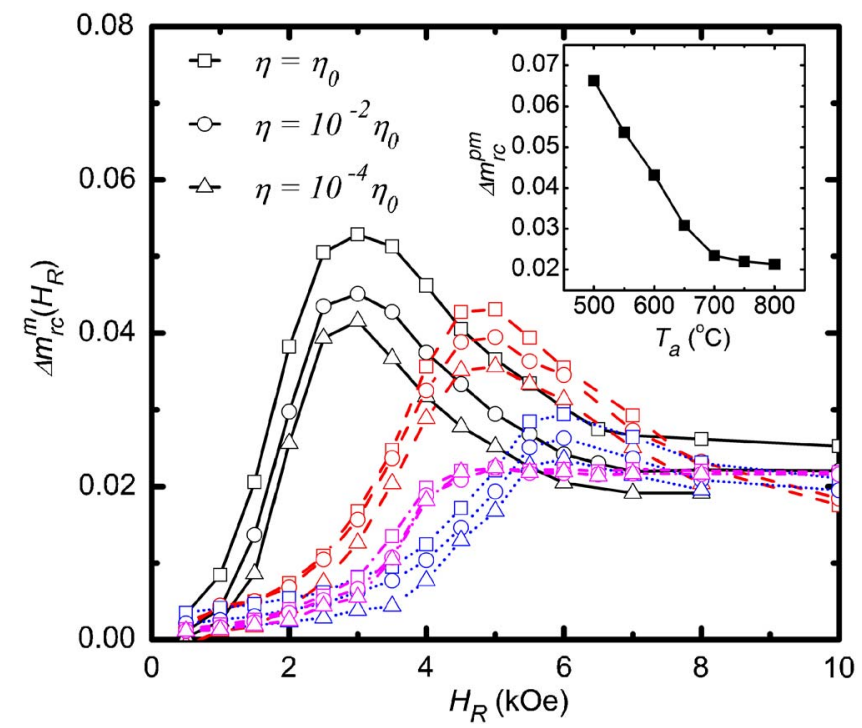

FIG. 3. (Color online) The dependence of $\Delta m_{\mathrm{rc}}^{m}\left(H_{R}\right)$ on the maximum applied field $H_{R}$ for 550 (black), 600 (red), 650 (blue), and $800{ }^{\circ} \mathrm{C}$ (purple) annealed samples with different sweep rates. The inset gives the dependence of $\Delta m_{\mathrm{rc}}^{\mathrm{pm}}$ on the annealing temperature.

$700-800{ }^{\circ} \mathrm{C}$, as shown in Fig. 3. This result further confirms that the recoil loop openness is time-dependent especially in the case of a two-phase nanocomposite. The time-dependent (sweep-rate-dependent) magnetization is a process connected with the thermal activation phenomena. ${ }^{16}$ To analyze the relation between $\Delta m_{\mathrm{rc}}^{m}\left(H_{R}\right)$ and the thermal fluctuation quantitatively, two different methods were used to measure the activation volume, i.e., the sweep-rate dependence of the coercivity $H_{c}(\eta)=H_{c}\left(\eta_{0}\right)+\left(k_{B} T / M_{s} V_{\mathrm{ac}}\right) \ln \left(\eta / \eta_{0}\right)$, and magnetic viscosity measurements $V_{\mathrm{ac}}=k_{B} T X_{\mathrm{irr}} / M_{s} H_{f} S$, respectively. ${ }^{15-18}$ Here $k_{B}, H_{f}, S$ and $\chi_{\text {irr }}$ are the Boltzmann constant, fluctuation field, magnetic viscosity parameter, and irreversible susceptibility, respectively. We compared the activation volumes obtained by these two methods and got similar results. Figure 4 shows the dependence of normalized coercivity $H_{c}(\eta) / H_{c}\left(\eta_{0}\right)$ on the sweep rate of the applied

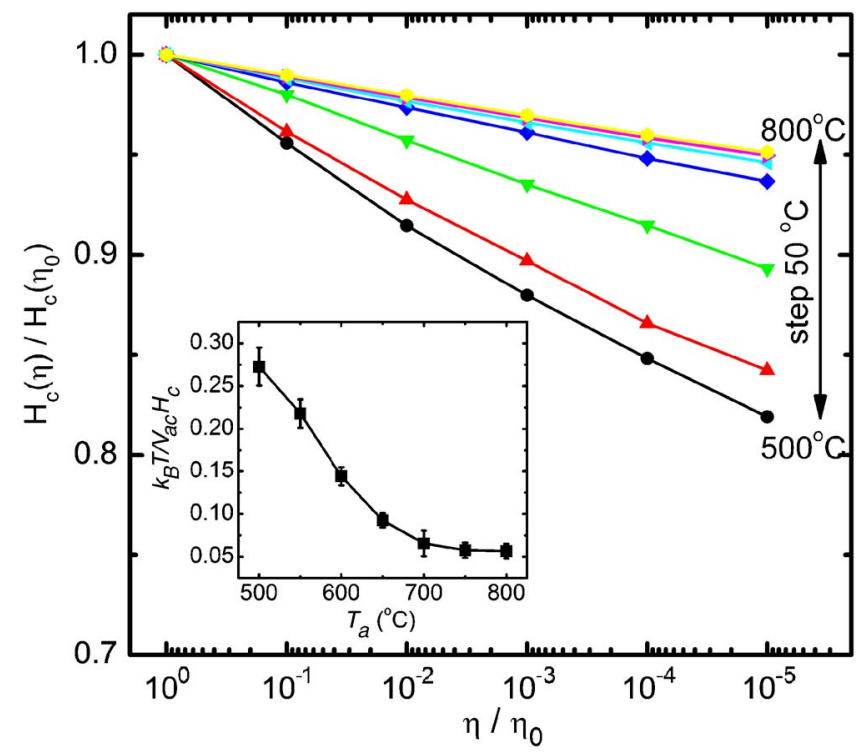

FIG. 4. (Color online) The dependence of coercivity on sweep rate for different samples. The inset gives the dependence of $k_{B} T / V_{\mathrm{ac}} H_{c}$ on the annealing temperature. 


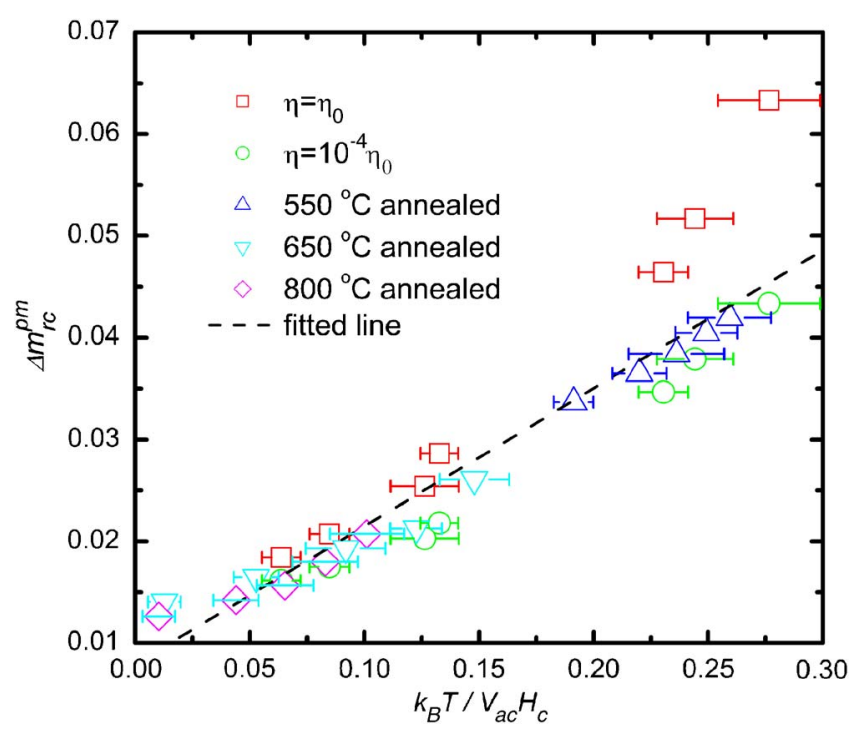

FIG. 5. (Color online) The dependence of $\Delta m_{\mathrm{rc}}^{\mathrm{pm}}$ on $k_{B} T / V_{\mathrm{ac}} H_{c}$ for different samples and different sweep rates. The square and circle symbols represent the data of the samples annealed at $500-800{ }^{\circ} \mathrm{C}$ for $\eta=\eta_{0}$ and $\eta=10^{-4} \eta_{0}$, respectively. The up triangle, down triangle, and diamond represent the SQUID data obtained at different temperatures for 550,650 , and $800^{\circ} \mathrm{C}$ annealed samples, respectively.

field as an example. It was found that the coercivity decreases fast with decreasing $\eta$ for the low-temperature annealed samples while slowly for the high temperature annealed samples. The inset gives the dependence of the slope, i.e., $k_{B} T / V_{\mathrm{ac}} H_{c}$, on the annealing temperature. One can see that $k_{B} T / V_{\mathrm{ac}} H_{c}$ decreases fast when $T_{a} \leqslant 650{ }^{\circ} \mathrm{C}$ and slowly when $T_{a}>650{ }^{\circ} \mathrm{C}$. Surprisingly, the shape of the $k_{B} T / V_{\mathrm{ac}} H_{c} \sim T_{a}$ curve is quite similar to that of the $\Delta m_{\mathrm{rc}}^{\mathrm{pm}}$ $\sim T_{a}$ curve as shown in the inset of Fig. 3 .

One sees from the insets of Figs. 3 and 4 that there is a close correlation between $\Delta m_{\mathrm{rc}}^{\mathrm{pm}}$ and $k_{B} T / V_{\mathrm{ac}} H_{c}$. Figure 5 shows the dependence of $\Delta m_{\mathrm{rc}}^{\mathrm{pc}}$ on $k_{B} T / V_{\mathrm{ac}} H_{c}$ for different sweep rates of the applied field. The square and circle symbols represent the data of the samples annealed at $500-800{ }^{\circ} \mathrm{C}$ for $\eta=\eta_{0}$ and $\eta=10^{-4} \eta_{0}$, respectively. It was found that $\Delta m_{\mathrm{rc}}^{\mathrm{pm}}$ increases with the increase of $k_{B} T / V_{\mathrm{ac}} H_{c}$. In addition, a rough linear relation between $\Delta m_{\mathrm{rc}}^{\mathrm{pm}}$ and $k_{B} T / V_{\mathrm{ac}} H_{c}$ was also observed. To confirm this correlation, $\Delta m_{\mathrm{rc}}^{\mathrm{pm}}$ and $V_{\mathrm{ac}}$ of 550,650 , and $800^{\circ} \mathrm{C}$ annealed samples were measured by a SQUID magnetometer at temperatures ranging from 5 to $390 \mathrm{~K}$. It was found that the fitting line is consistent with the measured data, as shown in Fig. 5.

In a hard/soft nanocomposite, the magnetic moments in the soft phase are exchange coupled with the moments in the hard phase. The magnetic moments in the soft-phase therefore tend to be unstable under a moderate reverse field and are easy to thermally reverse, especially for those in the center of soft grains due to the lower energy barrier compared to those near the hard phase. ${ }^{4,19}$ The unstable moments are affected sensitively by thermal fluctuation and contribute to the large openness in the recoil loops of the nanocomposite magnets. It should be noted that intergrain exchange coupling also induces unstable magnetic moments near the grain boundaries in a single-phase nanocrystalline hard magnet, ${ }^{4,20,21}$ and thus open recoil loops [see the $\Delta m_{\mathrm{rc}}^{m}\left(H_{R}\right)$ curve of the $800{ }^{\circ} \mathrm{C}$ annealed sample in Fig. 3]. However, large openness is normally observed in the recoil loops of nanocomposite magnets compared with single-phase magnets since the magnetic moments in the soft phase of nanocomposites are more unstable than those in grain boundary regions in single-phase hard magnets. The reason that the openness in the recoil loops decreases with increasing annealing temperature may be due to the decrease of soft-phase content, as seen in Fig. 3.

In summary, it has been revealed that recoil loop openness is sweep-rate dependent for the FePt-based nanocomposite and single-phase magnets obtained by annealing of the chemically synthesized $\mathrm{FePt} / \mathrm{Fe}_{3} \mathrm{O}_{4}$ nanoparticles. The nanocomposite systems show a larger recoil loop openness and stronger sweep-rate dependence than those of single-phase magnets. The quantitative analysis shows a linear relation between the recoil loop openness and the reciprocal activation volume, indicating that the recoil behavior is strongly related to thermal fluctuation.

This work is supported by US DoD/MURI Grant No. N00014-05-1-0497. This work is also supported by Center of Nanostructured Materials and Characterization Center for Materials and Biology at the University of Texas at Arlington.

${ }^{1}$ E. F. Kneller and R. Hawig, IEEE Trans. Magn. 27, 3588 (1991).

${ }^{2}$ R. Skomski and J. M. D. Coey, Phys. Rev. B 48, 15812 (1993).

${ }^{3}$ C. B. Rong, H. W. Zhang, X. B. Du, J. Zhang, S. Y. Zhang, and B. G. Shen, J. Appl. Phys. 96, 3921 (2004).

${ }^{4}$ C. B. Rong, H. W. Zhang, R. J. Chen, S. L. He, and B. G. Shen, J. Magn. Magn. Mater. 302, 126 (2006).

${ }^{5}$ E. H. Feutrill, P. G. McCormick, and R. Street, J. Phys. D 29, 2320 (1996)

${ }^{6}$ D. Goll, M. Seeger, and H. Kronmuller, J. Magn. Magn. Mater. 185, 49 (1998).

${ }^{7}$ A. Bollero, O. Gutfleisch, K.-H. Muller, L. Schultz, and G. Drazic, J. Appl. Phys. 91, 8159 (2002).

${ }^{8}$ C. L. Harland, L. H. Lewis, Z. Chen, and B. M. Ma, J. Magn. Magn. Mater. 271, 53 (2004).

${ }^{9}$ K. Kang, L. H. Lewis, J. S. Jiang, and S. D. Bader, J. Appl. Phys. 98, 113906 (2005).

${ }^{10}$ C. B. Rong, H. W. Zhang, B. G. Shen, and J. P. Liu, Appl. Phys. Lett. 88, 042504 (2006).

${ }^{11}$ C. B. Rong, V. Nandwana, N. Poudyal, Y. Li, J. P. Liu, Y. Ding, and Z. L. Wang, J. Phys. D 40, 712 (2007).

${ }^{12}$ Y. Choi, J. S. Jiang, J. E. Pearson, S. D. Bader, and J. P. Liu, Appl. Phys. Lett. 91, 022502 (2007).

${ }^{13}$ G. S. Chaubey, V. Nandwana, N. Poudyal, C. B. Rong, and J. P. Liu, Chem. Mater. 20, 475 (2008).

${ }^{14}$ H. Zeng, J. Li, J. P. Liu, Z. L. Wang, and S. H. Sun, Nature (London) 420 , 395 (2002).

${ }^{15}$ R. Skomski, R. D. Kirby, and D. J. Sellmyer, J. Appl. Phys. 93, 6820 (2003).

${ }^{16}$ R. Street, J. C. Wooley, and P. P. Smithh, Proc. Phys. Soc. London, Sect. B 65, 679 (1952).

${ }^{17}$ J. W. Harrell and A. F. Khapikov, J. Appl. Phys. 87, 5708 (2000).

${ }^{18}$ B. A. Jones, J. D. Dutson, K. O’Grady, B. J. Hickey, D. Li, N. Poudyal, and J. P. Liu, IEEE Trans. Magn. 42, 3066 (2006).

${ }^{19}$ M. K. Griffiths, J. E. L. Bishop, J. W. Tucker, and H. A. Davies, J. Magn. Magn. Mater. 234, 331 (2001).

${ }^{20}$ C. B. Rong, H. W. Zhang, X. B. Du, J. Zhang, S. Y. Zhang, and B. G. Shen, J. Magn. Magn. Mater. 277, 221 (2004).

${ }^{21}$ R. Fischer and H. Kronmuller, J. Magn. Magn. Mater. 191, 225 (1999). 